\title{
A revised mental health classification for use in general medical settings: the ICD11-PHC
}

\author{
David Goldberg
}

Chairman, WHO Advisory Group for Classification in Primary Care, email David.Goldberg@iop.kcl.ac.uk

\begin{abstract}
The World Health Organization (WHO) was aware that many hospital doctors and general practitioners did not use the detailed ICD-10 classification of mental and behavioural disorders (WHO, 1993), which had been produced for mental health professionals, and so it commissioned a specially modified version suitable for general medical settings. The new system was required to have modified - but not exact - equivalence to the main classification, and to consist of clinical descriptions rather than operational criteria for each of the proposed categories. The system would describe typical presenting complaints for each category in this setting, as well as the diagnostic features and the differential diagnosis for each disorder. An important new feature of the system was that it included the information that should be given to the patient and family, described the effective psychological and drug treatments and gave indications for specialist referral.

The 26 conditions recommended by a group consisting of psychiatrists and general practitioners (GPs) is given in Box 1, together with the corresponding $F$ (or $Z$ ) number for the main classification (Ustun et al, 1995; WHO, 1996):
\end{abstract}

\section{Experience with the ICD10-PHC}

A study by Upton et al (1999) with established GPs showed that the guidelines had no impact on the overall detection of mental disorders, the accuracy of diagnosis or the prescription of antidepressants, but there was a significant increase in the number of patients diagnosed with depression or unexplained somatic symptoms, and the GPs also made increased use of psychological interventions. A well-conducted randomised controlled tiral by Croudace et al (2003) with established GPs similarly failed to show that the guidelines had any impact either on detection or on patient outcomes. However, the ICD10-PHC has had a major impact in lowand middle-income countries, and is used in the training of nurses and multi-purpose health workers, as well as medical officers (Jenkins et al, 2002).

However, some of the ICD10-PHC disorders were equivalent to existing categories in the parent classification, and did not take into account developments in diagnostic thinking. An interesting example of this concerns 'medically unexplained symptoms', which appear to have fallen out of favour with our GP colleagues, who have taken the view that even some medically explained symptoms can be abnormally prolonged and accentuated. Psychiatrists have taken a similar view: the new concept of 'complex somatic symptom disorder' being field tested for DSM-V also draws attention not to whether somatic symptoms can be explained, but

\author{
Box 1 The 26 conditions included in ICD10-PHC \\ F00 Dementia \\ F05 Delirium \\ F10 Alcohol use disorder \\ F11 Drug use disorder \\ F17.1 Tobacco use disorder \\ F20 Chronic psychosis \\ F23 Acute psychosis \\ F31 Bipolar disorder \\ F32 Depression \\ F40 Phobic disorders* \\ F41 Panic disorder \\ F41.1 Generalised anxiety \\ F42.2 Mixed anxiety and depression* \\ F43 Adjustment disorder* \\ F44 Dissociative disorder \\ F45 Unexplained somatic complaints* \\ F48 Neurasthenia* \\ F50 Eating disorders \\ F51 Sleep problems \\ F52 Sexual disorders (male and female) \\ F70 Mental retardation \\ F90 Hyperkinetic disorder \\ F91 Conduct disorders \\ F98 Enuresis \\ Z63 Bereavement* \\ *Not to be included in ICD11-PHC.
}

to the cognitive components that may accompany them, whether they are part of a known physical disease or not.

'Mixed anxiety depression disorder' (MADD) was introduced in ICD-10 in order to take account of the fact that patients may just miss the diagnostic threshold for either generalised anxiety or depressive episode, but if they have symptoms of both disorders they are often distressed and disabled by them. However, it is an unsatisfactory concept because there is an unbroken continuum between such 'subthreshold' patients and others who are above the threshold for both, and are at present (confusingly) described as being 'comorbid' for two quite separate disorders. This is because mood disorders and anxiety disorders are in two different chapters of the parent ICD. A revised classification needs to take account of these patients with a combination of symptoms, who give the most common presentation of psychological distress in general medical practice, as well as often being severely disabled by their symptoms.

\section{A fresh look at the problem}

The ICD11-PHC is currently under development; the process is advised by a group consisting of approximately equal numbers from high-income, and low- and middle-income 
countries, of primary care physicians and psychiatrists who actually teach mental health skills to trainees in primary care, and of men and women. The deputy chairman is $\mathrm{Dr}$ Michael Klinkman, a GP who represents WONCA (the World Organization of National Colleges, Academies and Academic Associations of General Practitioners/Family Physicians), and another member, Dr Marianne Rosendal, is the European representative on WONCA where classification is concerned.

In our early discussions, many of the disorders in ICD10$\mathrm{PHC}$ are recommended to be retained - often with suitable amendments - but there have been several interesting new disorders suggested, as well as several disorders proposed for removal. Perhaps the most radical proposal is to abandon the distinction between anxiety disorders and mood disorders, and to gather them all under the single umbrella of 'dysphoric disorders'. Within this important group, two innovations are proposed. First, some simple operational criteria will be tested in field trials to assess whether clinicians in the field find them useful; if they do not, we could return to diagnosis by descriptions of clinical prototypes. Even if they do like the operational criteria, we will need to recalibrate the point on the scale equivalent to what was previously described as MADD. The simple scales will allow a clinician to diagnose depression and anxiety on their own, or the combination of both - to be called 'anxious depression'. Second, where any of these three disorders achieve the severity required for a 'case', any somatic symptoms not part of a known physical disorder will be assumed to be related to the dysphoric disorder. Those whose symptoms fall short of the requirements for any of these three diagnoses, but who are distressed and disabled by their current symptoms (whether dysphoric or somatic), are to be given the residual diagnosis of 'distress disorder'. Distress disorder replaces a motley collection of minor disorders, including neurasthenia (or chronic fatigue) and adjustment disorder.

The concept of anxiety disorder will not be exactly equivalent to generalised anxiety disorder (GAD), which by definition has to last at least 6 months. Clinicians in general medical practice need to know what is wrong with the patient now, rather than forming a lifetime concept of the patient's psychological health. Current anxiety is very much more common than GAD, and needs to be recognised if the patient is to receive appropriate reassurance and support.

A new category called bodily distress disorders will include conversion disorder (fairly common in some lowerincome countries), health preoccupation (a new disorder similar to hypochondriasis) and the less severe 'bodily distress syndrome'. In the syndrome, the patient is both distressed and concerned and has three or more somatic symptoms in one bodily system. This is diagnosed only if the patient does not have one of the three dysphoric disorders.

Post-traumatic stress disorder and panic/agoraphobia are other new adult disorders. In addition, the GPs on the group wish to have a single category of personality disorder, equivalent to borderline personality. These patients are well known to GPs, and we will try the concept out in a field trial.

Bereavement has been deemed to be surplus to requirements because it is not the only transition that is followed by a psychological disturbance.

Tobacco use disorder has been retained because of its public health importance, and the fact that patients may ask GPs for advice on how to reduce their use of tobacco.
Box 2 The 28 disorders to be field tested for ICD11-PHC

Childhood disorders

1 Intellectual development disorder (was mental retardation)

2 Autism spectrum disorder (new)

3 Specific learning disability (new)

4 Attention-deficit hyperactivity disorder (ADHD)

5 Conduct disorder

6 Enuresis, encopresis

Psychotic disorders

7 Acute psychosis

8 Chronic psychosis

9 Bipolar disorder

Dysphoric disorders

10 Anxious depression (new)

11 Depressive disorder

12 Anxiety disorder

13 Distress disorder (replaces F42.2, F43, F48)

14 Post-traumatic stress disorder (PTSD) (new)

15 Panic/agoraphobia (was panic disorder)

Body distress disorders

16 Bodily distress syndrome (new - was unexplained somatic complaints)

17 Health preoccupation (new)

18 Conversion disorder (was dissociative disorder)

Bodily function disorders

19 Sexual function disorder, male

20 Sexual function disorder, female

21 Sleep disorder

22 Eating disorder

Substance use disorders

23 Alcohol use disorders

24 Drug use disorders

25 Tobacco use disorders

Personality disorder

26 Borderline personality (new)

Acquired neurocognitive disorders

27 Dementia

28 Delirium

Two new childhood disorders are autism spectrum and specific learning disabilities, as it is thought important that GPs recognise them. They are part of the list of childhood disorders being drawn up by the Childhood Disorders Group at the $\mathrm{WHO}$, and they will be field tested with all the other categories to see whether GPs recognise them and find them useful. The 28 disorders to be field tested have been arranged in eight rough groups, shown as Box 2.

These proposals are radical indeed, and by no means all of the proposed disorders will survive the field tests. Each proposed category will be commented upon by experts who are not part of the group, as well as by the main advisory group responsible for ICD-11. Final amendments will be made by the primary care group before the revised classification is released for field tests. The field tests are likely to be quite extensive, and to involve studies in both high-income and low- and middle-income countries. A second set of revisions will be made after the field tests. Disorders that survive the field tests must have an equivalent disorder in the main classification - a requirement which may cause a problem with the new concept of anxious depression, since 
it requires some modification to the meta-structure of diagnoses used in the main classification.

The field tests will at first be confined to the diagnostic classification to be used in primary care; discussion about optimal management has been deferred to a later stage, but is likely to use the forms of management recommended by the mhGAP study (WHO, 2008), with possible additional headings.

\section{References}

Croudace, T., Evans, J., Harrison, G., et al (2003) Impact of the ICD-10 Primary Health Care (PHC) diagnostic and management guidelines for mental disorders on detection and outcome in primary care: cluster randomised controlled trial. British Journal of Psychiatry, 182, 20-30.
Jenkins, R., Goldberg, D. P., Kiima, D., et al (2002) Classification in primary care: experience with current diagnostic systems. Psychopathology, 35, 127-131.

Upton, M. W., Evans, M., Goldberg, D. P., et al (1999) Evaluation of ICD-10 PHC mental health guidelines in detecting and managing depression within primary care. British Journal of Psychiatry, 175, 476-482.

Ustun, T. B., Goldberg, D. P., Cooper, J., et al (1995) New classification for mental disorders with management guidelines for use in primary care: ICD-10 PHC chapter five. British Journal of General Practice, 45, 211-215.

WHO (1993) The ICD-10 Classification of Mental and Behavioural Disorders. World Health Organization.

WHO (1996) Diagnostic and Management Guidelines for Mental Disorders in Primary Care: ICD-10 Chapter V Primary Care Version. Hogrefe \& Huber.

WHO (2008) mhGAP: Mental Health Gap Action Plan. Scaling Up Care for Mental, Neurological and Substance Use Disorders. World Health Organization.

\section{Policy on mental health}

\section{David Skuse}

Behavioural and Brain Sciences Unit, Institute of Child Health, London, UK, email dskuse@ich.ucl.ac.uk

O ur theme this month concerns nascent psychiatric services in countries that are still developing their mental health provisions, but which face specific and diverse challenges. The most dramatic example of this is Iraq, where there continues to be far more conflict, corruption and instability than is ever reported in the Western media. Over $85 \%$ of non-governmental organisations have stopped operating in Iraq in recent years, and the future is uncertain for those that remain. Dr AlObaidi writes about the impact of the recent conflict on the mental health of children living in this traumatised country. There are concerns about the chances of creating a stable country in the future, when the current generation of children become adult, unless something is done to address their needs now. There are no formally trained child and adolescent psychiatrists, and it is not clear how the author's plea for a comprehensive and culturally sensitive child and adolescent mental health service could be answered in the near future without financial and professional assistance from outside Iraq itself.

Dr Araya and colleagues discuss a different challenge, in Chile. The aim was to find a way of providing good-quality mental healthcare within the primary care sector. Chile is one of those countries in South America with a burgeoning economy. Its growth rate, in terms of gross domestic product, was $4.3 \%$ in 2010 , on a par with Mexico. There is an enthusiasm for innovation, and funding is available to make it happen. Over the past 20 years several studies within Chile have examined the prevalence of psychiatric disorder in the general population. Interestingly, it seems that the impact of these 'home-grown' investigations, supported by the Ministry of Finance, has been far greater than that of innovations derived from studies in countries with a stronger scientific infrastructure. The authors describe clearly the steps taken to implement and evaluate the intervention, which provides a paradigm for countries aiming to establish novel psychiatric services that do not simply imitate the European/ US out-patient model.

Finally, Dr Osei and colleagues discuss the issue of mental health legislation in Ghana, a country that has fewer active psychiatrists now than in 2003. Existing services follow a traditional format, with their foundations set in large psychiatric hospitals. They have a relatively high ratio of admission to attendance. As in most other African countries, Ghana's mental health legislation is outdated and outmoded in both its scope and its application. Fortunately, a new mental health act has been drafted. One of the key aims will be, as in Chile, to move resources into the community and away from centralised in-patient care. We have previously, within International Psychiatry (vol. 4, no. 4, October 2007), discussed the important role of traditional healers in Africa and the need for psychiatric services to establish a dialogue with them. It has been estimated that there are no fewer than 45000 such healers in Ghana, and monitoring of their activities will be subsumed under the new legislation. Unfortunately, for a variety of reasons the authors discuss, bringing the act into force has proved more problematic than they had anticipated. 